\title{
The Design and Research of Theoretical Experiment Evaluation and System Based on Higher Learning
}

\author{
Shuhua Bai, Suling Li , Haidong Yang \\ Nanchang Institute of Technology, Nanchang, Jiangxi, 330044, China
}

Keywords: College, Theoretical Experiment Evaluation, Innovation System.

\begin{abstract}
In recent years, the development of institutions of higher learning has been very rapid, the laboratory construction funds have been large and the laboratory hardware facilities have been significantly improved. How to evaluate for the benefit of the people, how to give full play to the laboratory in talents cultivation, scientific research and social service function, further enhance the vitality of the laboratory, optimizing the allocation of resources, realize the resource sharing, improve the quality of experiment teaching and scientific research level, effectively raise the quality of laboratory teaching and become the important research topic in the college lab and equipment management. It is of great significance to improve the experimental innovation and evaluation of universities and to realize the goal of talents training in colleges and universities.
\end{abstract}

\section{Introduction}

As an important part of school teaching, experimental teaching has an important role to play in cultivating students' innovative thinking and innovation ability. The scientific evaluation of the teaching quality of the laboratory will combine the evaluation results with the laboratory's investment. It is an effective means to promote the quality improvement of experimental teaching in colleges and universities. Purport of this research by using system dynamics theory to carry on the beneficial attempt in this aspect, the comprehensive efficiency in laboratory, the effect of experimental teaching, scientific research and technology development and the social service, integrated management and the evaluation index system, promote the development of comprehensive experimental teaching.

\section{Theoretical Experiment Evaluation and System Analysis of Colleges and Universities}

The system considers new ways of thinking about the whole operation of teaching people to improve the "group intelligence" of the overall operation of human organizations. When people are faced with a complex problem is always used to split it into can handle moments to think, and then consolidate, such thinking is necessary, but it makes people easy to overlook more insight into the overall form factor - the whole interaction between molecules and the formation of the complex phenomena, the theory is called "dynamic complex". Sometimes it can be offset by an individual or group to improve all the problems, and most of the dynamic complex in the process of slow gradient action and results, there is a time gap between the theory called the "time lag". This process is extremely difficult to detect, even if it is not too late to know how to handle effectively. One organization lacks systematic thinking, and the more the employee tries to solve the problem, the worse the long-term consequences are because of the wrong direction. Why does this happen? Because you can't see the system behind it. The idea of systematic thinking holds that structures influence behavior, even if very different people, when they are in the same system, tend to produce similar results. System structure is referring to with the passage of time affect the behavior of some key variables, the relationship among people in a structure are usually all can't see how these structure operation, only to find himself had to do so, but the small perturbation of a variable in the system would through the whole system and take action and escalating. Therefore, through the study and application of systems thinking, find out the structural reasons behind the problem of complex, and by changing the structure and improving behavior change form is an important and 
creative work.

At present, the research of experimental teaching evaluation system has a lot of, common evaluation theory and method of data envelopment analysis method, matter-element analysis method, grey correlation evaluation method, artificial neural network analysis method, analytic hierarchy process (ahp) and fuzzy evaluation method, etc. After study compared the various evaluation methods, the author thinks that, apply any kind of single evaluation method can completely solve the problem of the experiment teaching quality evaluation, use most of these studies is the analytic hierarchy process (AHP) and fuzzy evaluation method.

In addition, the laboratory evaluation index system, the qualitative index of the proportion is larger, the fuzzy evaluation method is now is a effective method to evaluate the qualitative indicators, so, using the fuzzy evaluation method to analyze the qualitative part of the laboratory evaluation system more. Dan-ping liu teacher of hohai university in June 2003, 9 (2) of the journal acta in jiangsu teachers university of technology published in the journal of experimental teaching quality evaluation system of research and practice, using the fuzzy mathematical method has carried on the statistical analysis, the result of the experiment teaching assessment and evaluation system is established.

These evaluation methods are mostly based on the evaluation indicators (factors) is the basis of mutual independence of this assumption, but in fact, some evaluation indexes (factors) is the mutual independence between relative, but the correlation is absolute; How to fully tap the internal information between evaluation index, maximum performance of nonlinear characteristics of the mutual influence between the factors, make the review results more scientific and reasonable, is a complex system to assess an important theoretical research direction in the field of research, this article is using the complex dynamic feedback analysis, system dynamics method for this problem did some preliminary study and discussion, put forward the basic ideas and methods of the evaluation index system.

\section{Overview of Research and Analysis Methods}

As early as the 1950s, western developed countries began to pay attention to the evaluation of research and development work, mainly from the level of science and technology and management level to evaluate the laboratory work. Into one end of the 20th century, the evaluation of the laboratory work has entered a new stage, some experts and scholars tried to put some of the advanced evaluation theory and method is introduced into the laboratory evaluation, such as the explanation structure model method to determine evaluation index, the weights were determined by analytic hierarchy process (ahp), and through computer software, evaluation, etc., but has yet to form a complete system.

Evaluation work of the laboratory in China began in the 1980 s, the country, the industry (department) and local provincial science and technology management department by adopting a supporting institution annual appraisal, in the form of peer experts appraisal and quantitative data collection and evaluation. Experiment teaching quality assessment in the $90 \mathrm{~s}$ began to become an important task of college teaching evaluation, especially with the improvement of people about the importance of the experiment, people began studying the experimental teaching quality evaluation system, and have produced many good evaluation method.

Methods of Quantification. The determination of the assessment system is first reference to national laboratory evaluation method of the institutions of higher learning basic course "make sure the principle of evaluation system, then it is concluded that assessment of primary and secondary indexes and the evaluation items, then adopts the direct quantitative in mathematics and second quantization and refinement of decomposition method of statistical processing of data to determine the weight coefficient of every index. Finally, the evaluation system is used to evaluate experimental teaching. Shu Ming teacher fire using indicators principles put forward a set of teaching evaluation index system and evaluation of quantitative table, to establish the quality standard of experimental teaching and the requirements of each teaching link, teaching management, teaching process, teaching staff are given equipment, laboratory equipment, teaching reform, the 
teaching effect of six level index, 31 secondary index and 47 points, involves each main link of experiment teaching. Learn shun) of east China university of science and technology exhibition in countries studied under the guidance of evaluation index system of experimental courses in the whole teaching plan after the position, function and characteristics of the relation between the scientific nature and feasibility, teaching conditions, process and the relationship between the teaching effect, evaluation and the relationship between reform, and it is concluded that by 4 first-level indicators, 11 second-level indicators of evaluation system, then using quantitative mathematical methods to obtain grade standards, and evaluation standards. Li huixian of ningbo university and liu jianmin of Shanghai jiao tong university have adopted similar methods.

Analytic Hierarchy Process. The evaluation index system of laboratory comprehensive benefit is an organic whole, and the impact of each index on the evaluation results is different. In order to obtain scientific and accurate evaluation results, the weights of indicators need to be determined. Many researchers use analytic hierarchy process to calculate weights. Analytic hierarchy process (ahp) is associated with the decision scheme of element is decomposed into goals, principles, level, on the basis of the qualitative and quantitative analysis of the decision-making method, which are set according to the number 1 a 9 scale of judgment matrix, under the condition of satisfy the consistency check, the eigenvectors corresponding to the largest eigenvalue is a layer of elements on the level relative to the weight vector. The key to calculate weight is to determine the judging matrix. Ma Sheng lamp of zhejiang normal university teacher starting from the necessity of university basic course teaching laboratory comprehensive benefit evaluation, this paper discusses the laboratory four principles of comprehensive benefit evaluation index system design (objectivity principle, orientation principle, feasibility principle and comparability principle), constructed with 4 first-level indicators, 21 secondary indicators of basic course laboratory comprehensive benefit evaluation index system, index weight calculation, analytical hierarchy process (ahp) at all levels of the judgment matrix. The weight coefficient was determined by the same method in huangjincheng and shegang [6] of guangxi university, and then the data processing was carried out using the central compression treatment (minus the mean and the standard deviation).

Comprehensive Analysis Method. Many analysis method on the evaluation system, no single evaluation method can completely solve the problem of comprehensive evaluation of key laboratory, northwestern polytechnical university Zhang Yiting [8], consider an evaluation method for the combined method. After evaluating the actual research of the laboratory, comparing the application scope of the analytic hierarchy process and the fuzzy evaluation method, the two methods are used to evaluate. Them on the basis of investigation and research, the application of modern comprehensive evaluation theory and method, puts forward the comprehensive evaluation on the concept of national defense science and technology key laboratory, emphatically probes into the comprehensive evaluation index system and evaluation standard, and expounds the fuzzy comprehensive evaluation method was used to assess the implementation of the national defense science and technology key laboratory procedures, and finally, evaluation explained with an example of actual operation process. This method combines the advantages of the first two methods and is able to analyze the evaluation index system from quantitative and qualitative aspects.

Above methods all have, index quantification method is qualitative index system of the experiment are analyzed, and rely more on the role of the existing system of indicators to make weight coefficient judgment, lack of quantitative analysis, this method analysis closely enough, that is to say no to demonstrate the effective mathematical methods, Analytic hierarchy process (ahp) is a kind of index system of currently used for the experimental analysis of the most commonly used method, and fuzzy rules can be better to deal with data results, although the two methods can comprehensively from the aspects of qualitative and quantitative analysis of a system, but this kind of evaluation methods are mostly based on the evaluation indicators (factors) is the basis of mutual independence of this assumption, but in fact, some evaluation indexes (factors) is the mutual independence between relative, but the correlation is absolute; How to fully tap the internal information between evaluation index, maximum performance nonlinear characteristic of the mutual influence between the factors and make evaluation result more scientific and reasonable, is a 
complex system to assess an important theoretical research direction in the field of study.

Evaluation Method Based on Complex System. Object in the face of large, complex systems, such as the construction of colleges and universities in the assessment of teaching quality evaluation, laboratory construction, large in the study of system simulation credibility evaluation, etc., in the face of numerous information necessary for the inductive analysis, system evaluation occupies the important status and role, it is the scientific basis of decision.

The research areas of complexity science are mainly complex and complex systems, typical of social economic system and its complexity. The study of complex system and system complexity can help people understand the development rules and motivation of the system, so as to better adapt and control the system. As one of the five big school of international complexity science research, System Dynamics, the System Dynamics, SD) is the most effective tools for the study of complex systems and complexity, it according to the theory of ordinary differential equations as the main tool of social and economic System for a class of high order time, such as multiple feedback loop, highly nonlinear complex System of the combination of qualitative and quantitative research. In particular, the system dynamics starts from the microstructure of the complex system and constructs the basic feedback structure of the system to simulate and analyze the dynamic behavior of the system.

\section{Conclusion}

The establishment of the experimental evaluation system is an important link in the construction and management of national defense science and technology laboratory. The development of modern evaluation theory provides a rich technique and method for laboratory evaluation. In the laboratory life cycle, the exploration of the updated thinking and the technical methods comprehensively analyze the laboratory performance factors, and the establishment of a scientific evaluation system has been the subject of many experts and scholars. This paper aims to put forward a new experimental quality evaluation method, it is using system dynamics theory and method in combination with Peter $\mathrm{m}$. Senge system thinking management technology to establish a fundamental mode, the system more clearly reflect intuitive experiments the relationship between the indexes, and through the established evaluation system reflect the quality of the experimental teaching so as to better promote the development of teaching, to cultivate more talents to create conditions.

\section{Acknowledgements}

Fund Project: This paper is the 2015 annual Jiangxi Province Department of Education College of Humanities and social science research project, project number: JY1574, the name of the project: "the study on the theory of higher education evaluation and innovation experimental system stage series of research results. Subject number: GL15101 project name: a series of research results based on the innovation of e-commerce service model in urban community informatization.

\section{References}

[1] Shu fire, et al. Research on experimental teaching of higher learning, journal of hainan normal university, 2002, 1:4-8

[2], Shun et al., experimental course assessment programme, laboratory research and exploration, 2001, 20 (6) : 37-39

[3], Li huixian, China's higher education evaluation index system problems and countermeasures [J], higher science education, 2003, (4) : 27-30.

[4] Liu Jianmin, preliminary evaluation of experimental teaching quality [J], laboratory research and exploration, 2002, 21 (3) : 4-8 
[5] Ma Sheng lamp, etc., the first exploration of comprehensive benefit evaluation of higher teaching laboratory, journal of zhejiang normal university, 2004, 2:199-202

[6] Zhang Jiangong, college lab management mode selection and innovation development, journal of south China university of technology, 2005, 7 (5) : 78-80

[7] Wei Shaobo, the simulation evaluation of laboratory evaluation work, journal of guangxi national college, 2004, 10 (2) : 105-108

[8] Zhang Yi-Ting, et al. Research on comprehensive evaluation system of key laboratory of defense science and technology [J], laboratory research and exploration, 2005, 24 (4) : 90-98 